\title{
Physics at the Planck time
}

Superstring theory, perhaps the most notorious attempt to unify general relativity with quantum mechanics, was once described as a piece of twenty-first-century physics that fell into the twentieth century. Certainly, physicists struggling to marry these reluctant partners have long sensed that the crucial piece of the puzzle has yet to be invented. But a theory of quantum gravity remains more a question of tidiness than anything else - quantum effects on spacetime become important only on time and length scales well out of experimental reach. A quantum field theory of electromagnetism has plenty of practical relevance for applied physics, and such a field theory for the nuclear forces generates no shortage of testable predictions. But gravity yields to the quantum rod only at the so-called Planck scale: over distances of about $10^{-33} \mathrm{~cm}$ and times of $10^{-43} \mathrm{~s}$.

A physics to match the Planck timescale is the biggest challenge to physicists in the coming century. This is the

timescale relevant to the graviton, the putative carrier of the gravitational force. And naturally enough, only such a theory can extend our understanding of the Big Bang inside the first $10^{-43}$ seconds of existence.

What will a theory of quantum gravity do to space-time? Will it be like John Wheeler's 'quantum foam', furiously alive with ephemeral black holes and worm holes? Or will it be more like Abhay Ashtekar's fabric of woven loops? Will particles become strings, and will the strings be supersymmetric? How many extra dimensions will turn up, curled out of sight over the Planck distance?

And will we ever be able to test such a theory? One quantum-gravity researcher has confessed that "it seems highly unlikely that a machine will ever be built with which these minute distances can be studied directly". For guidance, it seems that physicists may have to fall back on the old stand-bys: elegance and beauty.

Philip Ball

\section{The speed of computers}

One version of Moore's Law, predicted by Intel co-founder Gordon Moore in 1965, has it that computer chips double in speed every 18 months. Nothing moves any faster, of course - the chips simply become more powerful by packing more devices into the same space. Speed is about miniaturization. Recent research on the ultimate size limits for silicon technology have fuelled speculation about whether Moore's Law is headed for a crash. By 2012, at the current rate of shrinkage, transistors will have become so small that the silicon oxide films will no longer guarantee adequate insulation between conducting regions: they become switches that cannot be fully turned off. What happens then to our expectation that the next generation of machines will be better, meaner and faster?

All manner of factors determine how quickly, say, the Nature web page downloads onto your screen, many of them scarcely connected to the component density on the chips inside the processor. In some parts of the world it can take ten minutes or more. Yet you would be waiting a lot longer if the data were streaming down old copper telephone cables rather than glass optical fibres. Fibre-optic networks have a greater carrying capacity that speeds up transmission by one or two orders of magnitude relative to electrical lines. But that is only a fraction of what should be possible with photonic technology.

And the electronics at either end are soon to be stretched to their limit to cope with the transmission speeds that photonics offers. Gigabit-per-second distributed networks have already been demonstrated, and laser diodes can in principle blink out 100

Gbits per second; but electronics lose the ability to cope at half that rate. All-optical networks will then be required, and networks working at 100 Gbits per second are already in development. At some point, the light will need to go on a chip, in photonic integrated circuits.

But photonics is an embryonic technology, and plagued with obstacles in comparison with mature electronics. No clear rival to the transistor has yet emerged from this or any other direction, such as single-electron devices and molecular electronics. So perhaps we will have to adjust our expectations as the information revolution reaches a plateau. Moore predicts that we have about two generations of computers left with current technologies, and "beyond that, life gets very interesting".

Philip Ball

\section{Lifespan extension}

Eat more fibre! Consume less cholesterol! Drizzle more olive oil! Have fewer children later in life! Drink a glass of red wine a day! And so it goes on. Rarely a day goes by without the emergence of a new 'theory' in that most seductive realm of scientific advances — lifespan extension.

Chromosome structure hints that our cells have genetic 'fuses' that mete out their allotted time. Antibiotics, vaccinations and antiseptics have brought about hikes in life expectancy in the developed world which have raised the average lifespan to almost double that at the last fin de siècle — nigh-on 80 years and still rising — as this century draws to a close. The intractability of several cancers such as Hodgkin's lymphoma and leukaemia has been tamed and many others look to follow soon; the hard nut of cognitive decline is on the verge of being cracked with drugs and neuron replacement; and workable synthetic organs and xenotransplants are around the corner.

But the current obsession with all things genetic, molecular, high-tech and expensive risks missing the point that both the World Bank and World Health Organization (WHO) make patently clear. That is, for the majority of the planet the most powerful lifespan-extending technologies of all - good, basic public health and preventative medicine - are still a far-off dream, even if they are, scientifically speaking, old hat.

As we usher in the third millennium, the WHO estimates that the citizens of the world's poorest countries (who make up two-fifths of the global population) have almost half the life expectancy at under 50 years - of those living in the richest nations. And that gap is widening, not narrowing, often in both directions. One third of the planet's population do not have secure access to safe water or sufficient food, let alone drugs, immunizations, or even the simplest health education. Elsewhere, billions of dollars are being spent teasing out the intricacies of gene therapy and memory enhancement. Addressing this inequity is surely one of the greatest and most pressing challenges facing us all, if not as academics, then as people. 